\title{
A novel PCR method targeting staphostatin genes differentiates Staphylococcus aureus from Staphylococcus epidermidis in clinical isolates and nasal microbiome samples
}

\section{Marina Zoheir Ghattas}

Department of Microbiology and Immunology, Faculty of Pharmacy, Cairo University, Cairo, Egypt Marwa Tarek ElRakaiby ( $\nabla$ marwaelrakaiby@gmail.com )

Department of Microbiology and Immunology, Faculty of Pharmacy, Cairo University, Cairo, Egypt https://orcid.org/0000-0002-6508-7536

Ramy Karam Aziz ( $\sim$ raziz1@gmail.com )

Department of Microbiology and Immunology, Faculty of Pharmacy, Cairo University, Cairo, Egypt https://orcid.org/0000-0002-4448-7100

Hamdallah H. Zedan

Department of Microbiology and Immunology, Faculty of Pharmacy, Cairo University, Cairo, Egypt

\section{Method Article}

Keywords: microbiome, molecular diagnostics, Gram-positive cocci, bioinformatics, 16S rRNA

Posted Date: August 28th, 2019

DOI: https://doi.org/10.21203/rs.2.13709/v1

License: (1) (i) This work is licensed under a Creative Commons Attribution 4.0 International License.

Read Full License 


\section{Abstract}

Staphylococcus aureus is a pathogen associated with community-acquired and healthcare-associated infections, and is known for rapid acquisition of resistance to multiple antibiotics. Staphylococcus epidermidis is another staphylococcal species commonly seen as a commensal, but may cause opportunistic infections. These two closely related species may naturally co-colonize and inhabit the human skin and nose; thus it is important to develop methods to differentiate between them. Conventional differentiation methods (e.g., mannitol salt agar, coagulase test, Analytical Profile Index kits) require culturing and isolating the bacteria, while molecular methods based on polymerase chain reaction (PCR) or sequencing are culture independent, highly accurate, and sensitive. They may be objectively interpreted and can be adapted for high-throughput assays. Here, we aimed to develop a rapid and accurate method to differentiate mixtures of $S$. aureus and $S$. epidermidis in clinical isolates and microbiome samples. Using comparative genomics tools (e.g., MetaRef and PATRIC bioinformatics resource center), we compared the core and pan genomes of $S$. aureus and $S$. epidermidis to determine signature genes for each species. Among candidate genes, we chose the virulence genes, staphostatin $B$ and staphostatin $A$ as genetic biomarkers for $S$. aureus and $S$. epidermidis, respectively. We designed PCR primers that target specific sequences within each gene and tested them on DNA isolated from laboratory strains, clinical isolates, nasal isolates, and nasal swabs. Our developed method is simple, specific, sensitive, and can be applied to metagenomic samples as well as competition or co-evolution studies in which the two organisms are co-cultured.

\section{Introduction}

Staphylococcus aureus is a major human nasal colonizer inhabiting about one third of the world's population (Yan et al. 2013). It is the causative agent of several healthcare-associated infections such as pneumonia, meningitis, endocarditis, and osteomyelitis. Healthcare-associated infections represent a global life-threatening problem, especially in developing countries. They lead to financial losses by extending the duration of hospitalization (5-29.5 days in developing countries (Allegranzi et al. 2011)), by diminishing the productivity of human resources, and by expanding the burden of treatment cost on governments. More importantly, healthcare-associated infections often lead to the emergence of multiresistant pathogens, popularly known as superbugs. The World Health Organization (WHO) reported that ten out of every 100 surgical site infections (the second dominating type among hospitalized patients) are caused by S. aureus (Allegranzi et al. 2011).

Although primary infections by S. aureus are not deadly in nature, this pathogen causes several complications, many of which are invasive, and is one of the main causes of sepsis, which leads to death if not treated properly. Thus, the emergence of multiresistant strains makes this pathogen one of the most dangerous bacteria. In fact, S. aureus is one of the so-called ESKAPE pathogens, which are declared as a global public health threat (Pendleton et al. 2013). Patients infected with methicillin-resistant $S$. aureus (MRSA) are $64 \%$ more likely to die than those infected with non-resistant $S$. aureus (World Health Organization 2018). 
Staphylococcus epidermidis is another staphylococcal species that predominantly colonizes the axillae, head, and nares of humans. It is a major skin resident and constitutes about $90 \%$ of aerobic resident microbiota (Cogen et al. 2008; Sanford and Gallo 2013), but may cause opportunistic infections, especially to patients using indwelling medical devices.

1. aureus and S. epidermidis share morphological characteristics and genotypic traits, and are practically indistinguishable on microscopic examination. The differentiation between these two closely related staphylococcal species mainly depends on culture-based methods. For example, $S$. aureus is able to ferment mannitol producing an acidic byproduct, which lowers the $\mathrm{pH}$ of the mannitol salt agar (MSA) medium and turns its color into yellow, while S. epidermidis cannot ferment mannitol. S. epidermidis belongs to the group of coagulase-negative staphylococci (CoNS), which are distinguished from $S$. aureus by the absence of the coagulase enzyme. For years, selective culture media, e.g., MSA and Baird Parker agar, and biochemical tests, e.g., the coagulase test and many tests in Analytical Profile Index (API) kits have been playing an important role in their differentiation.

Although these methods give accurate results in most instances, they may fail to differentiate between the two species when present in mixed cultures or clinical specimens. For example, $S$. aureus strains isolated from nasal swabs were reported to produce false-negative results on MSA (dos Santos et al. 2015). The major confirmatory test for $S$. aureus, the coagulase test, may also provide negative results (Kateete et al. 2010). Moreover, all these tests require fresh overnight cultures, and are thus time consuming.

Consequently, there is a continuous need for cost-effective, more rapid and reliable molecular tools for identification and differentiation of these two common staphylococcal species, especially when identification is performed directly without culture. Accordingly, a few culture-independent methods were developed (Chiang et al. 2012; Ghebremedhin et al. 2008; Hirotaki et al. 2011), but none of them was used on human microbiome samples. Here, we aim to develop a novel biomarker to allow the rapid molecular differentiation between $S$. aureus and $S$. epidermidis without the need for isolation or culture, notably in nasal swabs intended for microbiome analysis.

For this purpose, we used comparative genomics to find suitable markers, staphostatin $A$ in S. epidermidis and staphostatin B in S. aureus, and developed a PCR method, which proved to be sensitive and specific in discriminating both bacterial species either from pure isolates or in mixtures.

\section{Materials And Methods}

\section{Ethics statement}

All experiments were conducted according to the national and international ethical standards. The experimental protocols, including sample collection from nasal swabs and consent forms, were revised 
and approved by the Faculty of Pharmacy Cairo University Safety and Health Ethics Committee (Protocol approval \#MI1137 on 23/6/2014).

\section{Bioinformatics and comparative genomics analysis}

We compared the core genomes of S. aureus and S. epidermidis using MetaRef (Huang et al. 2014), a comparative genomics tool, and we used PATRIC Proteome Comparison Service (Antonopoulos et al. 2017; Wattam et al. 2014) to compare the predicted proteomes of the two bacterial species. Ribosomal sequences were aligned with Clustal Omega (Sievers and Higgins 2014), available at the European Bioinformatics Institute's website (see Internet Resources).

\section{Microbiome simulation experiments}

Simulation experiments were designed to examine the accuracy of different $16 \mathrm{~S}$ amplicon classification strategies in resolving mixtures of closely related staphylococcal species. Four mixtures of 16S rRNA gene sequences of three staphylococcal species: S. aureus (genome accession IDs: NC_002745 and NC_007793), S. epidermidis (genome accession IDs: NC_004461 and NC_002976) and S. saprohyticus (genome accession ID: NC_007350) were computationally generated.

QIIME v. 1.9.1 (Caporaso et al. 2010) was used for operational taxonomic unit (OTU) classification from the mixtures, and two QIIME OTU-picking protocols were used (open and closed reference). Additionally, two ribosomal sequence databases were selected for taxonomical classification: Greengenes (DeSantis et al. 2006) and RDP (Cole et al. 2014).

\section{Collection of nasal samples}

We collected duplicate nasal swabs from 12 healthy volunteers living in Cairo, Egypt, including ten nurses working at a regional hospital in Qasr El-Ainy District, Cairo, Egypt. Their ages ranged from 25 to 42 years, and they included ten females and two males.

Volunteers were asked to gently rotate sterile cotton swabs wetted with $0.9 \%$ sodium chloride solution four times in both anterior nares. All volunteers were informed of the study goals, benefits, and potential risks and provided informed consent.

\section{Bacterial strains and biochemical tests}

Different $S$. aureus and S. epidermidis standard and clinical strains were used in this work for testing PCR. Standard strains were among those in the reference collection of the Department of Microbiology and Immunology, Faculty of Pharmacy, Cairo University, and they included S. aureus N315, ATCC33591, and USA200; and S. epidermidis ATCC12228 and ATCC1457. All clinical isolates used were previously identified, after removal of all patient-identifying information, and biobanked as part of the aforementioned department's collection. All strains were kept in Brain Heart Infusion broth supplemented 
with $20 \%$ glycerol and were subcultured in Brain Heart Infusion broth or on Brain Heart Infusion agar plates.

Nasal swabs were surface inoculated on $10 \%$ blood agar and incubated at $37^{\circ} \mathrm{C}$ for $48 \mathrm{hrs}$. Morphologically different colonies were subcultured on Brain Heart Infusion agar then the isolated colonies were distinguished on selective media, e.g., MSA. The different isolates were Gram-stained and examined under the microscope. For confirmation, isolates were analyzed by conventional biochemical methods, namely the catalase test (with $\mathrm{H}_{2} \mathrm{O}_{2}$ from Sigma, Taufkirchen, Germany), coagulase test (on fresh serum), and API Staph kits (Biomérieux, Craponne, France).

Bacterial isolates obtained from the nasal samples were stored at $-70^{\circ} \mathrm{C}$ in Brain Heart Infusion broth supplemented with $20 \%$ glycerol.

\section{DNA extraction, precipitation, and purification}

From colonies: Three to five bacterial colonies, from an overnight-cultured plate, were suspended in $100 \mathrm{ml}$ of deionized sterile water or in TRIS-EDTA (TE) buffer, boiled for $2 \mathrm{~min}$, and finally centrifuged for $1 \mathrm{~min}$. The supernatant served as a template for PCR amplifications and was aliquoted and stored at $-20^{\circ} \mathrm{C}$ when not in use.

From nasal swabs: DNA was extracted by previously published chemical methods (Sagar et al. 2014; Singh et al. 2013), with the following modifications: To enhance the cell lysis of Gram-positive bacteria, we used beads from the PowerSoil $\circledR^{\circ}$ DNA isolation kit (MoBio laboratories Inc., Carlsbad, USA) in Eppendorff tubes containing $1 \mathrm{ml}$ lysis buffer, and the beads were beaten in a MO BIO Vortex Genie® 2 Vortex (MoBio laboratories Inc., Carlsbad, USA) at 10,000 revolutions per minute for 30 seconds.

The extracted DNA was quantified, and its purity was checked in a P330 NanoPhotometer ${ }^{\circledR}$ spectrophotometer (Implen, München, Germany). Whenever 260:280 ratios were low (i.e., $\leq 1.6$ ), the DNA was precipitated by the standard ethanol precipitation protocol (Green and Sambrook 2012).

For DNA sequencing, bands from PCR products on agarose gels were excised with sterile scalpels and purified by the QIAGEN Gel Extraction kit (Qiagen, Hilden, Germany).

\section{Primer design}

The NCBI protein accession numbers of staphostatin A for S. epidermidis and staphostatin B for S. aureus were OBZ50474 and CEH26156.1, respectively. From the protein database record, we obtained the corresponding coding DNA sequence of their respective genes ( $s s p C$ and $\operatorname{ecp} B$ ), and confirmed the gene length by comparison to other open-reading frames using BlastX (Altschul et al. 1990). 
Before designing the primers, we aligned the nucleotide sequences of staphostatin $A$ to all those present in NCBI NR database to detect any variations in the nucleotide sequence of all $S$. epidermidis strains. We repeated the previous steps with staphostatin $B$ in $S$. aureus species. Finally, we designed primers (Table 1) for each gene using PrimerQuest ( $($ Integrated DNA Technologies (IDT), Inc., San Diego, CA, USA) and we checked the primers' properties using OligoAnalyzer ${ }^{\circledR}$ (IDT, San Diego, CA, USA).

\section{Polymerase chain reaction design and running conditions}

PCR amplification was performed in $12 \mu \mathrm{l}$ reaction buffer, including $1.5 \mathrm{mM} \mathrm{MgCl}_{2}(5 x$ Green GoTaq ${ }^{\circledR}$ reaction buffer, Promega, Madison, WI, USA); $200 \mu \mathrm{M}$ of deoxyribonucleoside triphosphate (Thermo Fisher Scientific, CA, USA); $1.25 \mathrm{U}$ of Taq DNA polymerase (Promega, Madison, WI, USA); $10 \mathrm{mM}$ of each primer and $2 \mu$ of DNA.

The amplification was performed in a Veriti ${ }^{\mathrm{TM}}$ 96-Well Thermal Cycler, according to the following steps: $94^{\circ} \mathrm{C}$ for $2 \mathrm{~min} ; 30$ cycles of $94^{\circ} \mathrm{C}$ for $30 \mathrm{~s}, 55^{\circ} \mathrm{C}$ or $62^{\circ} \mathrm{C}$ for $30 \mathrm{sec}$, and $72^{\circ} \mathrm{C}$ for $18 \mathrm{sec}$; finally, $72^{\circ} \mathrm{C}$ for $5 \mathrm{~min}$. The annealing step was performed at $55^{\circ} \mathrm{C}$ for staphostatin $\mathrm{A}$ primers and $62^{\circ} \mathrm{C}$ for staphostatin $B$ primers. In each run, a non-template control, consisting of the same PCR reaction mixture without a DNA template, was used.

After amplification, $3 \mu \mathrm{l}$ of the reaction mixture was analyzed by electrophoresis on a $1 \%$ agarose gel in Tris-Acetate-EDTA buffer at $140 \mathrm{~V}$ for $40 \mathrm{~min}$ and stained with ethidium bromide. PCR bands were detected and imaged by MultiDoc-lt ${ }^{\mathrm{TM}}$ Imaging system (UVP, Cambridge, UK), and their sizes were determined in comparison to a 100-bp DNA ladder (Solis Biodyne, Tartu, Estonia).

\section{DNA sequencing}

For PCR product validation, DNA purified from excised gel bands were directly sequenced by the corresponding primers (Macrogen, Seoul, Korea).

Additionally, universal primers for bacterial 16S rRNA were used to confirm the bacterial identity. The sequences of these primers are 8F 5'-AGAGTTTGATCCTGGCTCAG-3' and 1492R 5'GGTTACCTTGTTACGACTT-3' (James 2010; Kong et al. 2007).

\section{Table 1: Nucleotide sequence of staphostatin $A$ and staphostatin $B$ primers}




\begin{tabular}{llll}
\hline Species & Primer name & Primer Sequence & Amplicon Size \\
\hline \multirow{2}{*}{ S. epidermidis } & Staphostatin A-Fw & 5' GTTGGTTCCATACATTAGAAG 3' & $155 \mathrm{bp}$ \\
\cline { 2 - 3 } & Staphostatin A-Rev & 5' TCGGATTGTCTACATTACTT 3' & \\
\hline \multirow{2}{*}{ S. aureus } & Staphostatin B- Fw & 5' CGCGAAGTRCCAATACCT 3' & \multirow{2}{*}{$268 \mathrm{bp}$} \\
\cline { 2 - 3 } & Staphostatin B- Rev & 5' GACACAACMAAACTCACACAT 3' & \\
\hline
\end{tabular}

\section{Results}

\section{Limitation of microbiome analysis methods as shown by in silico community simulation}

The motivation of this study is the need to differentiate $S$. aureus from $S$. epidermidis in mixtures, especially in human nasal microbiome samples in which the two organisms may co-occur. This need was triggered by the reported inability of classifying most organisms to the species level, in general, and staphylococci, in particular, in nasal microbiome samples (Frank et al. 2010).

To confirm this reported observation, we aligned the full sequences of all copies of the16S rRNA gene from two representative $S$. aureus and two representative $S$. epidermidis genomes (Fig. S1). In addition, we used the 16S rRNA sequences of a third species, Staphylococcus saprophyticus, as a contro for variability in the 16S rRNA sequence within genus Staphylococcus.

The alignment demonstrated a high similarity between the 16S rRNA sequences of the three species. The few nucleotide differences were scattered around the full length of the sequence; however, there were long stretches of $100 \%$ identical sequences, for example: the first 70 nucleotides, a long stretch between nucleotides 661 and 1005, and another stretch from 1161-1265 (Fig. S1).

As common $16 \mathrm{~S}$ amplicon-based methods for microbiome analysis use short amplicons spanning variable regions among all prokaryotes (e.g., V1-V3, V3-V4 or V4-V5), these regions are not necessarily able to differentiate staphylococcal species (Frank et al. 2010). To further investigate this limitation, we computationally generated four virtual microbiomes, each containing an unequal mixture of fragments of the 16S rRNA genes of the three staphylococcal species aligned above. Using QIIME (a standard microbiome analysis software package (Caporaso et al. 2010)), we aligned and classified the sequences in the four mixtures to the species level, and compared them to the expected proportions of the three represented staphylococcal taxa (Fig. 1). Analysis results varied based on the database used (Greengenes or RDP) and the OTU-picking protocol (open or closed reference methods). Overall, all methods successfully detected a vast majority of staphylococcal sequences; however, the software was 
often unable to assign the appropriate species. In many instances, the species was not identified and in fewer instances the wrong species was assigned. The $S$. aureus-to-S. epidermidis ratios noticeably varied (Fig. 1), emphasizing the need of genetic markers markers other than the variable regions of the $16 \mathrm{~S}$ rRNA gene for either species.

\section{Comparative genomics}

An ideal PCR to differentiate $S$. aureus from $S$. epidermidis in mixtures should target a gene present in all known $S$. aureus strains and absent in all $S$. epidermidis strains, and vice versa. If the latter condition is not achievable, the designed primers should at least not falsely generate a product of the same size from $S$. epidermidis, and vice versa.

As a first step towards this goal, we compared the core genomes of $S$. aureus and $S$. epidermidis using MetaRef (Huang et al. 2014). The analysis identified 191 marker proteins in $S$. aureus but not in S. epidermidis cores, and 89 marker proteins in $S$. epidermidis, absent in $S$. aureus. From these proteins, we focused on those that are predicted as "signature" for the species: unique to a particular species in comparison to all other species of the same genus as per MetaRef terminology. We chose staphostatin B (family IMG ID: 646073942; gene code $=s s p C$ ) from the signature proteins of $S$. aureus and staphostatin A (family IMG ID: 647323015; gene code = ecpB) from the signature proteins of $S$. epidermidis. The functional similarity of both proteins made them a relevant differentiating pair for the two species, in addition to being virulence biomarkers.

After choosing these two biomarkers, we examined each of them against the pan genome of both organisms to confirm that each staphostatin protein is present in one species and substantially different in the other. To this end, we used BlastN and tBlastN on each gene and protein, respectively, against NCBI GenBank. When the nucleotide sequence of staphostatin B was aligned to all $S$. aureus genomes, homologs were detected in all of them with high similarity and coverage (99\%-100\% and 99-100\% coverage, with E-values ranging from $2 \times 10^{-167}$ to $8 \times 10^{-171}$ ), and these matches were reciprocal best hits suggesting orthology. Conversely, when the sequence of staphostatin $B$ was aligned to all $S$. epidermidis genomes, the Blast hits had quite a low coverage (7-19\%), and these hits did not match staphostatin $B$ of $S$. aureus on reciprocal Blast analysis.

Likewise, we repeated the same steps for staphostatin A. All analyzed S. epidermidis genomes had orthologous hits with high coverage (100\%) and high similarity (98\% - 100\%). On the other hand, no significant full-length genes were matched to staphostatin A, but only partial alignments were obtained ( $67 \%$ to $90 \%$ similarity over $12 \%$ to $65 \%$ coverage). None of the obtained hits matched S. epidermidis staphostatin A on reciprocal BlastN analysis. 
As a confirmatory step, we compared the predicted proteomes of two $S$. aureus reference strains (N315 and USA300) against the predicted proteome of S. epidermidis ATCC12228 genome using PATRIC (Wattam et al. 2014). This comparison confirmed the absence of staphostatin B from S. epidermidis. On the other hand, when we compared the predicted proteome of $S$. epidermidis against that of $S$. aureus USA300 (as a reference strain), we got a partial match between staphostatin $A$ and another hypothetical protein in S. aureus (with $34.1 \%$ identity over $78 \%$ coverage). The hypothetical protein of $S$. aureus USA300 has Locus Tag AZ30_10180 and PATRIC ID fig|1458279.3.peg.1985.

\section{Primer design and in silico validation}

We designed a primer pair for each of the two signature genes chosen and used BlastN to test each primer pair for sensitivity and specificity. For sensitivity, we aligned each primer pair with the complete set of available genomes from the species they are supposed to detect. The degenerate primers targeting staphostatin $B$ were aligned to all $S$. aureus genomes and those targeting staphostatin $A$ successfully matched all $S$. epidermidis genomes. Specificity was also confirmed, as none of the primer pairs had any BlastN hits (at a permissive E-value threshold of 10).

\section{PCR analysis of staphostatin A and staphostatin B in representative strains and clinical isolates of S. aureus and $S$. epidermidis}

We initially tested both primers on ten $S$. aureus and two $S$. epidermidis strains available in our laboratory collection (Fig. 2). These included reference strains for both S. aureus and S. epidermidis as detailed in the Materials and Methods section. Additionally, we tested the primers on 13 MRSA clinical isolates and 24 isolates from nasal swabs.

Finally, to exclude the probability of false positive or false negative results because of interference, we tested each set of primers on DNA extracted from both strains, as well as a mixture of $S$. aureus and S. epidermidis. PCR results were $100 \%$ accurate: the staphostatin A primers were specific to all $S$. epidermidis strains, and the staphostatin $B$ primers were specific to all $S$. aureus strains.

\section{PCR on staphylococcal isolates from nasal swabs}

After testing the assay on DNA from laboratory and clinical isolates, mostly subcultured from frozen glycerol stock, we set out to test it on freshly isolated bacteria from nasal swabs. Out of 12 nasal swabs, we obtained 24 pure individual bacterial isolates, which we identified by traditional tools as S. aureus $(n=9)$ or $S$. epidermidis $(n=15)$. The assay was able to detect the right species every time $(100 \%$ sensitivity), and there was no cross-reactivity (Table 2 and Fig. 3 ).

For additional confirmation of bacterial identity, we amplified a large segment spanning most of the 16S rRNA gene from representative $S$. aureus and $S$. epidermidis isolates, using universal primers, and we sequenced the amplified products, which confirmed their identity. 


\section{PCR on community DNA}

Having tested the PCR assay on bacterial isolates and mixtures, we moved to the main application of the assay, which is differentiating between the two staphylococcal species in microbial communities without the need of cultivating or isolating bacteria.

DNA was directly extracted from the 12 swabs that were not used in culture-based analysis and tested for amplifiability by universal primers targeting the 16S rRNA genes (James 2010). Initially, only six out of 12 DNA preparations yielded amplification products with these universal primers, while the other six failed, possibly because of some interfering chemicals. Thus, DNA was ethanol-precipitated and resuspended in nuclease-free water. All precipitated DNA preparations successfully yielded amplification products.

Both staphostatin A and staphostatin B primers gave positive PCR results with all 12 nasal samples (Fig. 4).

\section{Sensitivity and specificity of the developed assay}

The developed PCR method allowed discrimination between S. aureus and S. epidermidis strains isolated from nasal samples. Furthermore, the results of PCR performed on the isolates matched the identification results obtained by standard microbiological diagnostic tools (Table 1).

In addition, we successfully detected the presence of $S$. aureus using staphostatin B primers in all the 12 nasal samples, including the samples from which we could not isolate $S$. aureus by culture methods (five out of 12 nasal samples). On the other hand, staphostatin A primers gave a positive PCR with two nasal samples from which we could not isolate S. epidermidis strains (Table 2 and Fig. 5).

\section{DNA sequencing to confirm PCR specificity}

The specificity of our designed primers for staphostatin $A$ - and staphostatin $B$ - encoding genes was confirmed by comparison of the PCR product sequences against NCBI GenBank.

In some instances, a non-specific band of $\sim 400 \mathrm{bp}$. appeared in some instances with community DNA amplified by staphostatin B primers. This PCR product was sequenced, and the sequence matched the sucC gene, which encodes succinate-CoA ligase subunit beta in S. epidermidis (Table 3). However, this band was easily distinguishable on the gels because of its different electrophoretic mobility than that of the specific bands. 


\section{Discussion}

For decades, microbiologists have been developing a series of culture media and biochemical tests for systematically identifying bacterial species. These culture-based methods are valuable in identifying some bacteria to the species or even strain levels; however, in many instances it is a challenge to differentiate closely related bacteria or to identify them in a community.

Exploring new methods for accurate identification and differentiation of closely related bacterial species is a continuous and pressing need. Even when a reliable assay is established, the discovery of novel strains, or novel strain variants, may sometimes challenge the well-established methods. Additionally, there is a need for simple, rapid assays for special purposes, such as resolving commonly cohabitating groups of microbes or distinguishing species in microbial communities.

This work focused on the discrimination between the two closely related species, $S$. aureus and S. epidermidis, both of which colonize the human nasal cavity. The isolation of these two bacteria and their identification is pivotal for microbiological diagnosis and epidemiological monitoring, especially in a hospital context.

Surprisingly, in many instances, it was not possible in this work to distinguish $S$. aureus and S. epidermidis by the existing methods (e.g., culture on MSA or coagulase test), notably when the bacteria were isolated from nasal swabs. Our observation agrees with published reports. For example, coagulasenegative $S$. aureus strains have been isolated since 1988 (Woo et al. 2001). A recent study reported that the specificity and sensitivity of the common tests for $S$. aureus (culture on MSA, DNase, and tube coagulase tests) is higher when the three tests are used together ( $100 \% \mathrm{vs.} 75 \%$ if any test is performed alone (dos Santos et al. 2015)). While commercial identification systems, e.g., API, present an appealing alternative, they are not widely or routinely used in developing countries because of their high running cost and because of resource limitations.

On the other hand, molecular methods outweigh traditional identification methods in many aspects, including their higher sensitivity and specificity, speed, and-above all-their independence on bacterial isolation and enrichment before the actual tests are performed. Although cost is an issue with molecular methods, in general, in many cases the running cost is lower when the assays are routinely used. By eliminating intensive labor, and the need for several media and reagents, these methods cut the direct and indirect costs on the long term. The simplest and almost ubiquitously used molecular identification method at the time being is the polymerase chain reaction (PCR).

A few genes have been targeted for simultaneous detection and discrimination of $S$. aureus and S. epidermidis (Chiang et al. 2012;Hirotaki et al. 2011). These gene targets have either been used in combination or in the context of multi-pathogen preset real-time PCR assays. 
For the differentiation between the two Staphylococcus species targeted in this work, 16S rRNA, 23S rRNA, fib, hsp60, cpn60, tuf, femA, and nuc genes have been suggested (Baron et al. 2004; Ghebremedhin et al. 2008; Goh et al. 1997; Hirotaki et al. 2011; Sunagar et al. 2013). Despite the sensitivity and universality of $16 \mathrm{~S}$ rRNA gene sequencing in identifying many bacteria up to species level, the similarity between $16 \mathrm{~S}$ rRNA genes of $S$. aureus and S. epidermidis exceeds $98 \%$ (BlastN analysis and multiple alignment shown in Supplemental Fig. S1), and therefore 16S rRNA gene surveys, which rely on the partial sequencing of variable regions within the $16 \mathrm{~S}$ gene, cannot resolve most mixtures of the two staphylococci. Moreover, $16 \mathrm{~S}$ rRNA analysis requires amplicon sequencing, which adds time and cost to the assays (Saruta et al. 1995; Zakrzewska-Czerwinska et al. 1992).

In particular, the V3-V4 region, most commonly used in microbiome analysis, cannot fully differentiate staphylococcal species (Fig. 1 and S1). While this variable region may resolve S. aureus in most instances, it cannot differentiae S. epidermidis from other CoNS, and this differentiation is database dependent (Frank et al. 2010). We validated this differentiation through in silico simulation experiments, which confirmed the variability between different OTU-picking strategies and reference databases-even when the same software was used (Fig. 1).

Analyses based on partial 16S rRNA amplicons lead to both false-positive (misclassifications, i.e., assigning species that do not exist in the community) and false-negative results (unresolved species, i.e., limiting the results to the genus level and failing to determine the exact species).

For the PCR-based method developed here, we chose two previously unused marker genes: $s s p C$ and ecpB, encoding Staphostatin B in S. aureus and Staphostatin A in S. epidermidis, respectively. Both staphostatins are cytoplasmic inhibitors of their corresponding secreted cysteine proteases (staphopain A and B) and thus protect the cytosolic proteins against the proteolytic activity of these two proteases (Kantyka et al. 2011). Staphostatin B plays other roles in the regulation of $S$. aureus growth, the ability to form a biofilm, and the organization of peptidoglycan layer in the cell envelope (Shaw et al. 2005). In $S$. epidermidis, both the cysteine protease and its inhibitor are encoded within the same operon ecpAB, while in $S$. aureus, both the protease and its inhibitor are encoded in the same operon sspABC (MartínezGarcía et al. 2018).

We tested and performed our PCR analysis on DNA extracted from isolated colonies or directly from nasal swabs, while previously developed methods were usually tested on cultured bacteria only. No cross-reactivity was observed as we tried each primer pair on either species or on mixtures of both species. The PCR results for laboratory strains and isolated colonies were confirmed by the traditional biochemical tests (which usually work properly on isolated colonies in the absence of contamination). For further validation, we sequenced the 16S rRNA gene from representative isolates and confirmed their identity as well. It is to be noted that this sequencing of a near-full length amplicon of the 16S rRNA gene is different from the methods based on 16S rRNA amplicon libraries, commonly used in microbiome analysis, as the latter targets small fragments of the 16S rRNA gene. 
Even when non-specific products incidentally appeared on amplifying DNA from microbiome samples with high microbial burden, these products were of different sizes, which would not interfere with the 200 bp amplicon size of staphostatin amplicons.

Finally, the PCR method described here was more sensitive in detecting $S$. aureus in five nasal samples, from which this species was not detected by culture-based methods. This result can either be seen as a sign of higher sensitivity or as a possible false-positive amplification. However, preliminary microbiome analysis indicated that $S$. epidermidis was much more abundant than $S$. aureus in these five samples (manuscript in preparation). The probability of isolating $S$. aureus from nasal swabs was reported to drop tenfold if $S$. epidermidis counts were higher than $10^{5} \mathrm{CFU} / \mathrm{swab}$ (Lina et al. 2003), which might explain why $S$. aureus could not be isolated from these nasal swabs with higher ratios of S. epidermidis.

Future studies will address the epidemiological value of the proposed method by conducting largescale studies to screen patients in hospitals, health-care workers, and community members for carriage of either of the two staphylococcal species. Additionally, future experimentation may address the impact of excessive organic material on the assay, if conducted-for example-on samples contaminated with patient blood or excretions. Such details are not in the scope of this manuscript.

\section{Conclusions}

Our validated PCR-based method can be used for the differentiation between two closely related bacterial species, $S$. aureus and $S$. epidermidis, present in pure colonies or in clinical samples. Both staphostatin $A$ and staphostatin $B$ are good biomarkers for $S$. epidermidis and $S$. aureus, respectively. Accordingly, this method will help to overcome the difficulty of differentiating the two species in microbiome surveys, which pose a challenge to culture-based methods.

\section{References}

Allegranzi B, Bagheri Nejad S, Combescure C, Graafmans W, Attar H, Donaldson L, and Pittet D (2011) Burden of endemic health-care-associated infection in developing countries: systematic review and meta-analysis. Lancet 377:228-241. 10.1016/s0140-6736(10)61458-4

Altschul SF, Gish W, Miller W, Myers EW, and Lipman DJ (1990) Basic local alignment search tool. J Mol Biol 215:403-410. 10.1016/S0022-2836(05)80360-2 [doi] S0022-2836(05)80360-2 [pii] 
Antonopoulos DA, Assaf R, Aziz RK, Brettin T, Bun C, Conrad N, Davis JJ, Dietrich EM, Disz T, Gerdes S et al. (2017) PATRIC as a unique resource for studying antimicrobial resistance. Brief Bioinform. $10.1093 / \mathrm{bib} / \mathrm{bbx} 083$

Baron F, Cochet MF, Pellerin JL, Ben Zakour N, Lebon A, Navarro A, Proudy I, Le Loir Y, and Gautier M (2004) Development of a PCR test to differentiate between Staphylococcus aureus and Staphylococcus intermedius. J Food Prot 67:2302-2305.

Caporaso JG, Kuczynski J, Stombaugh J, Bittinger K, Bushman FD, Costello EK, Fierer N, Peña AG, Goodrich JK, Gordon JI et al. (2010) QIIME allows analysis of high-throughput community sequencing data. Nat Methods 7:335-336. nmeth.f.303 [pii] 10.1038/nmeth.f.303 [doi]

Chiang YC, Lu HC, Li SC, Chang YH, Chen HY, Lin CW, and Tsen HY (2012) Development of PCR primers and a DNA macroarray for the simultaneous detection of major Staphylococcus species using groESL gene. Foodborne Pathog Dis 9:249-257. 10.1089/fpd.2011.1007

Cogen AL, Nizet V, and Gallo RL (2008) Skin microbiota: a source of disease or defence? Br J Dermatol 158:442-455. BJD8437 [pii] 10.1111/j.1365-2133.2008.08437.x [doi]

Cole JR, Wang Q, Fish JA, Chai B, McGarrell DM, Sun Y, Brown CT, Porras-Alfaro A, Kuske CR, and Tiedje JM (2014) Ribosomal Database Project: data and tools for high throughput rRNA analysis. Nucleic Acids Res 42:D633-642. 10.1093/nar/gkt1244

DeSantis TZ, Hugenholtz P, Larsen N, Rojas M, Brodie EL, Keller K, Huber T, Dalevi D, Hu P, and Andersen GL (2006) Greengenes, a chimera-checked 16S rRNA gene database and workbench compatible with ARB. Appl Environ Microbiol 72:5069-5072. 10.1128/aem.03006-05

dos Santos DC, da Costa TM, Rabello RF, Alves FA, and de Mondino SS (2015) Mannitol-negative methicillin-resistant Staphylococcus aureus from nasal swab specimens in Brazil. Braz J Microbiol 46:531-533. 10.1590/s1517-838246220140179

Frank DN, Feazel LM, Bessesen MT, Price CS, Janoff EN, and Pace NR (2010) The human nasal microbiota and Staphylococcus aureus carriage. PLoS ONE 5:e10598. 10.1371/journal.pone.0010598

Ghebremedhin B, Layer F, Konig W, and Konig B (2008) Genetic classification and distinguishing of Staphylococcus species based on different partial gap, 16S rRNA, hsp60, rpoB, sodA, and tuf gene sequences. J Clin Microbiol 46:1019-1025. 10.1128/jcm.02058-07

Goh SH, Santucci Z, Kloos WE, Faltyn M, George CG, Driedger D, and Hemmingsen SM (1997) Identification of Staphylococcus species and subspecies by the chaperonin 60 gene identification method and reverse checkerboard hybridization. J Clin Microbiol 35:3116-3121.

Green MR, and Sambrook J (2012) Molecular cloning: a laboratory manual. 
Hirotaki S, Sasaki T, Kuwahara-Arai K, and Hiramatsu K (2011) Rapid and accurate identification of human-associated staphylococci by use of multiplex PCR. J Clin Microbiol 49:3627-3631. $10.1128 / \mathrm{jcm} .00488-11$

Huang K, Brady A, Mahurkar A, White O, Gevers D, Huttenhower C, and Segata N (2014) MetaRef: a pangenomic database for comparative and community microbial genomics. Nucleic Acids Res 42:D617-624. 10.1093/nar/gkt1078

James G (2010) Universal bacterial identification by PCR and DNA sequencing of 16S rRNA gene. In: Schuller M, Sloots TP, Holliday CL, G.S. J, and I.W.J. C, eds. PCR for Clinical Microbiology. New York: Springer, 209-214.

Kantyka T, Shaw LN, and Potempa J (2011) Papain-like proteases of Staphylococcus aureus. Adv Exp Med Biol 712:1-14. 10.1007/978-1-4419-8414-2_1

Kateete DP, Kimani CN, Katabazi FA, Okeng A, Okee MS, Nanteza A, Joloba ML, and Najjuka FC (2010) Identification of Staphylococcus aureus: DNase and Mannitol salt agar improve the efficiency of the tube coagulase test. Ann Clin Microbiol Antimicrob 9:23. 10.1186/1476-0711-9-23

Kong Y, Xia Y, Nielsen JL, and Nielsen PH (2007) Structure and function of the microbial community in a full-scale enhanced biological phosphorus removal plant. Microbiology 153:4061-4073. 10.1099/mic.0.2007/007245-0

Lina G, Boutite F, Tristan A, Bes M, Etienne J, and Vandenesch F (2003) Bacterial competition for human nasal cavity colonization: role of staphylococcal agr alleles. Appl Environ Microbiol 69:18-23.

Martínez-García S, Rodríguez-Martínez S, Cancino-Diaz ME, and Cancino-Diaz JC (2018) Extracellular proteases of Staphylococcus epidermidis: roles as virulence factors and their participation in biofilm. APMIS 126:177-185. 10.1111/apm.12805

Pendleton JN, Gorman SP, and Gilmore BF (2013) Clinical relevance of the ESKAPE pathogens. Expert Rev Anti Infect Ther 11:297-308. 10.1586/eri.13.12

Sagar K, Singh SP, Goutam KK, and Konwar BK (2014) Assessment of five soil DNA extraction methods and a rapid laboratory-developed method for quality soil DNA extraction for $16 \mathrm{~S}$ rDNA-based amplification and library construction. J Microbiol Methods 97:68-73. 10.1016/j.mimet.2013.11.008

Sanford JA, and Gallo RL (2013) Functions of the skin microbiota in health and disease. Semin Immunol 25:370-377. 10.1016/j.smim.2013.09.005

Saruta K, Hoshina S, and Machida K (1995) Genetic identification of Staphylococcus aureus by polymerase chain reaction using single-base-pair mismatch in $16 \mathrm{~S}$ ribosomal RNA gene. Microbiol Immunol 39:839-844. 
Shaw LN, Golonka E, Szmyd G, Foster SJ, Travis J, and Potempa J (2005) Cytoplasmic control of premature activation of a secreted protease zymogen: deletion of staphostatin B (SspC) in Staphylococcus aureus 8325-4 yields a profound pleiotropic phenotype. J Bacteriol 187:17511762. $10.1128 / \mathrm{jb} .187 .5 .1751-1762.2005$

Sievers F, and Higgins DG (2014) Clustal Omega, accurate alignment of very large numbers of sequences. Methods Mol Biol 1079:105-116. 10.1007/978-1-62703-646-7_6

Singh SP, Sagar K, and Konwar BK (2013) Strategy in metagenomic DNA isolation and computational studies of humic acid. Curr Res Microbiol Biotechnol 1:9-11.

Sunagar R, Deore SN, Deshpande PV, Rizwan A, Sannejal AD, Sundareshan S, Rawool DB, Barbuddhe SB, Jhala MK, Bannalikar AS et al. (2013) Differentiation of Staphylococcus aureus and Staphylococcus epidermidis by PCR for the fibrinogen binding protein gene. J Dairy Sci 96:28572865. 10.3168/jds.2012-5862

Wattam AR, Abraham D, Dalay O, Disz TL, Driscoll T, Gabbard JL, Gillespie JJ, Gough R, Hix D, Kenyon R et al. (2014) PATRIC, the bacterial bioinformatics database and analysis resource. Nucleic Acids Res 42:D581-591. 10.1093/nar/gkt1099

Woo PC, Leung AS, Leung KW, and Yuen KY (2001) Identification of slide coagulase positive, tube coagulase negative Staphylococcus aureus by $16 \mathrm{~S}$ ribosomal RNA gene sequencing. Mol Pathol $54: 244-247$.

World Health Organization (2018) Antimicrobial Resistance. Available at http://www.who.int/mediacentre/factsheets/fs194/en/ (accessed January, 25, 2018.

Yan M, Pamp SJ, Fukuyama J, Hwang PH, Cho DY, Holmes S, and Relman DA (2013) Nasal microenvironments and interspecific interactions influence nasal microbiota complexity and $S$. aureus carriage. Cell Host Microbe 14:631-640. 10.1016/j.chom.2013.11.005

Zakrzewska-Czerwinska J, Gaszewska-Mastalarz A, Pulverer G, and Mordarski M (1992) Identification of Staphylococcus epidermidis using a 16S rRNA-directed oligonucleotide probe. FEMS Microbiol Lett 100:51-58.

\section{INTERNET RESOURCES}

- Clustal Omega: version available at the European Bioinformatics Institute's website, URL: https://www.ebi.ac.uk/Tools/msa/clustalo (last accessed August 20 2019)

- PrimerQuest Program: Integrated DNA Technologies (IDT), URL: https://www.idtdna.com/PrimerQuest/ (last accessed August 20 2019-requires free sign in) 
- Oligo Analyzer Program: Integrated DNA Technologies (IDT), URL: https://www.idtdna.com/calc/analyzer (last accessed August 20 2019-requires free sign in)

- Blast: available at the NCBI website: URL: https://blast.ncbi.nlm.nih.gov/Blast.cgi (last accessed August 20 2019)

\section{Declarations}

\section{Financial disclosures:}

This work was funded by the annual research budget allocated by Cairo University to the Faculty of Pharmacy, Department of Microbiology and Immunlogy, without any external funding. The university research funding department does not interfere with study design, data collection or analysis, decision to publish, or manuscript preparation.

\section{Competing interests:}

Neither of the authors has any personal or financial competing interests to declare.

\section{Approvals:}

As indicated under "Materials and Methods", experimental protocols were revised and approved by the Faculty of Pharmacy Cairo University Safety and Health Ethics Committee (Protocol approval \#MI1137 on 23/6/2014).

\section{Tables}

Due to technical limitations Tables 2 and 3 are only available as a download in the supplementary section.

\section{Figures}




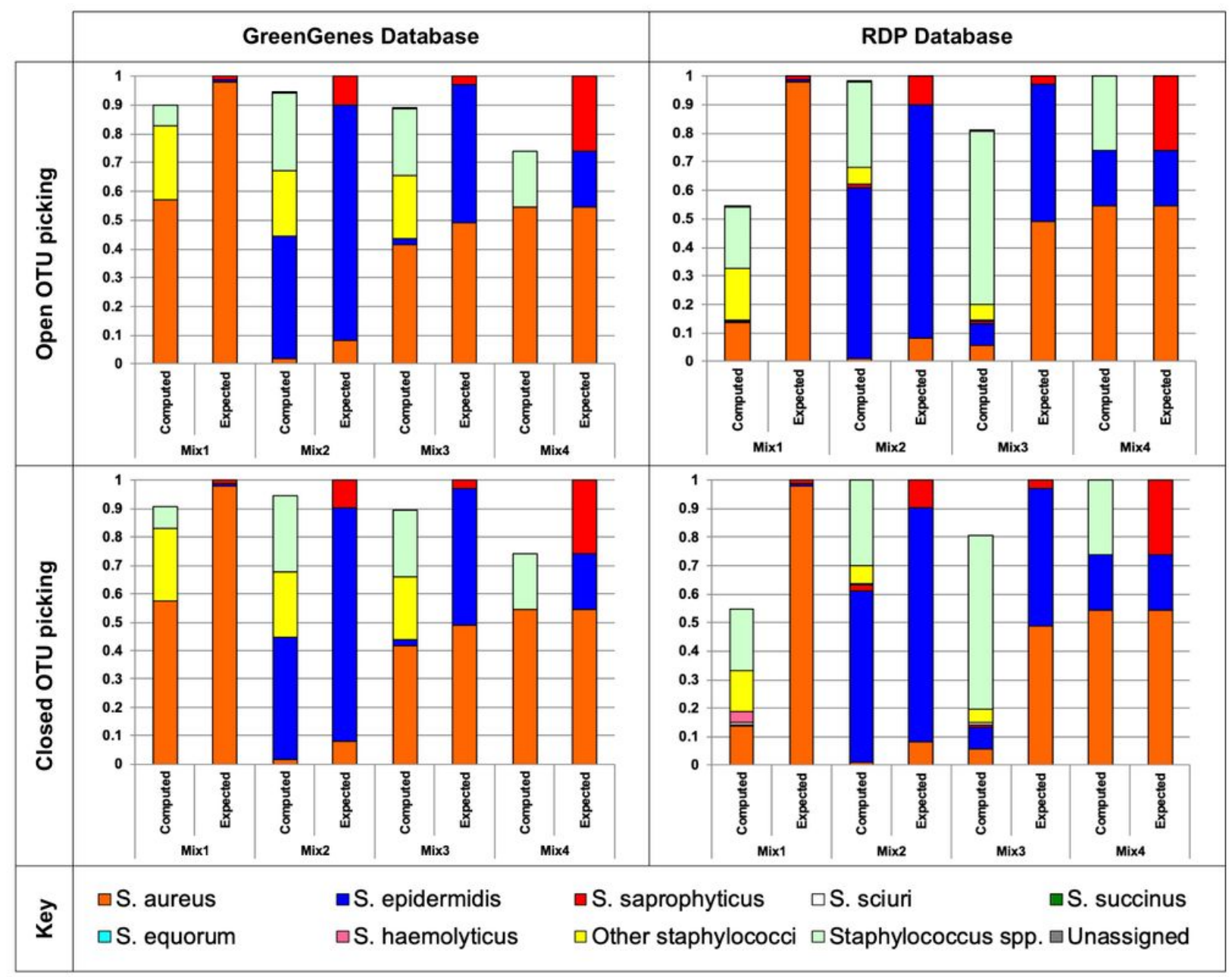

Figure 1

Results of simulation experiments. Stacked bar charts describe simulation experiments performed four different computationally generated communities containing mixtures of different proportions of $\mathrm{S}$. aureus, S. epidermidis, and S. saprophyticus. The mixtures were analyzed with two different OUT-picking strategies: open reference strategy (upper panels) and closed reference strategy (lower panels) and with two different 16S rRNA databases: Greengenes (left) and RDP (right). 


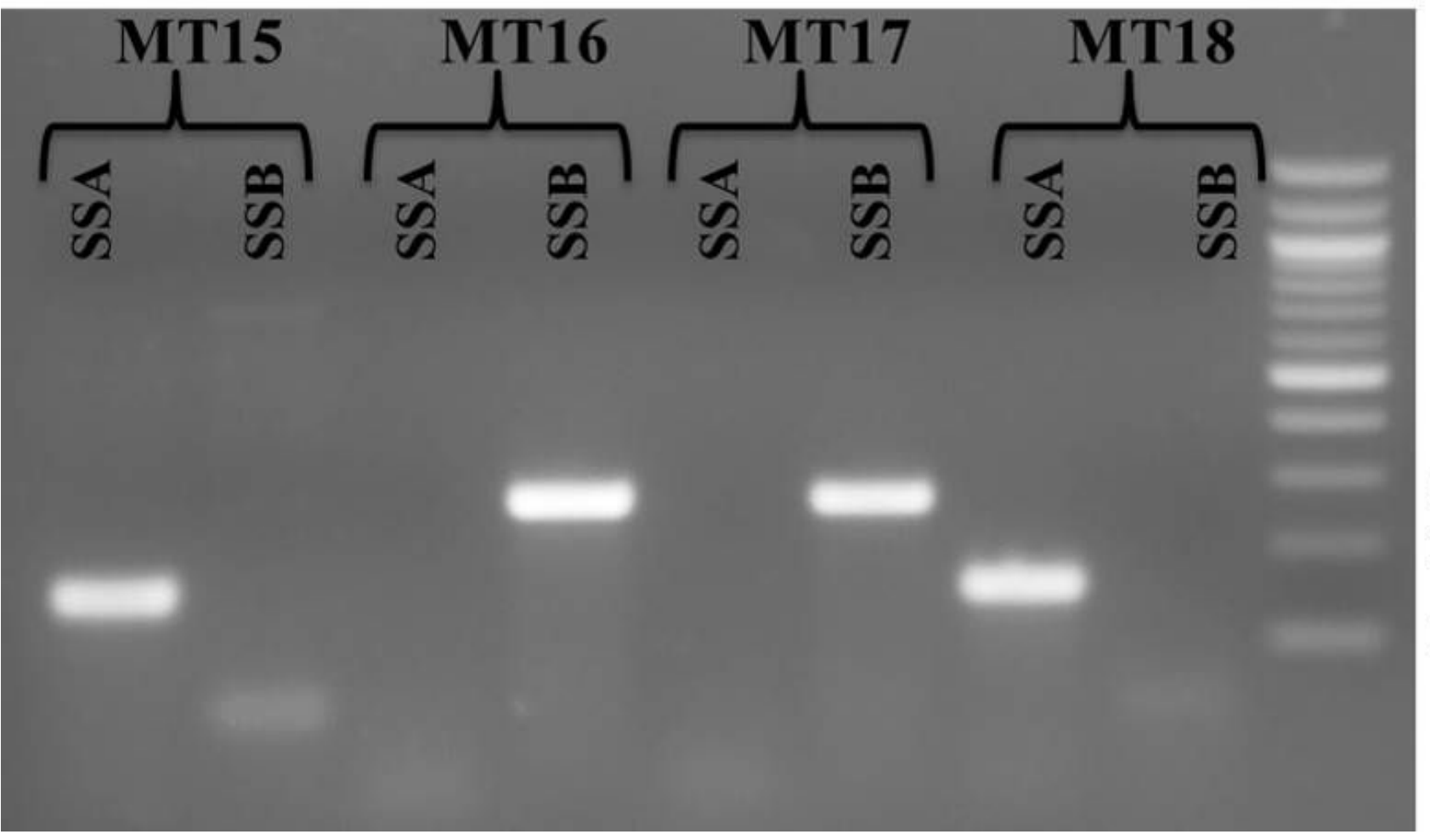

300 bp 200 bp 100 bp

\section{Figure 2}

Agarose gels showing the results of PCR for the identification of individual nasal isolates. PCR using staphostatin A and staphostatin B primers was performed on DNA extracted from nasal isolates. MT15 to MT18 are four representative cultured nasal isolates. SSA: staphostatin A primers; SSB: staphostatin B primers

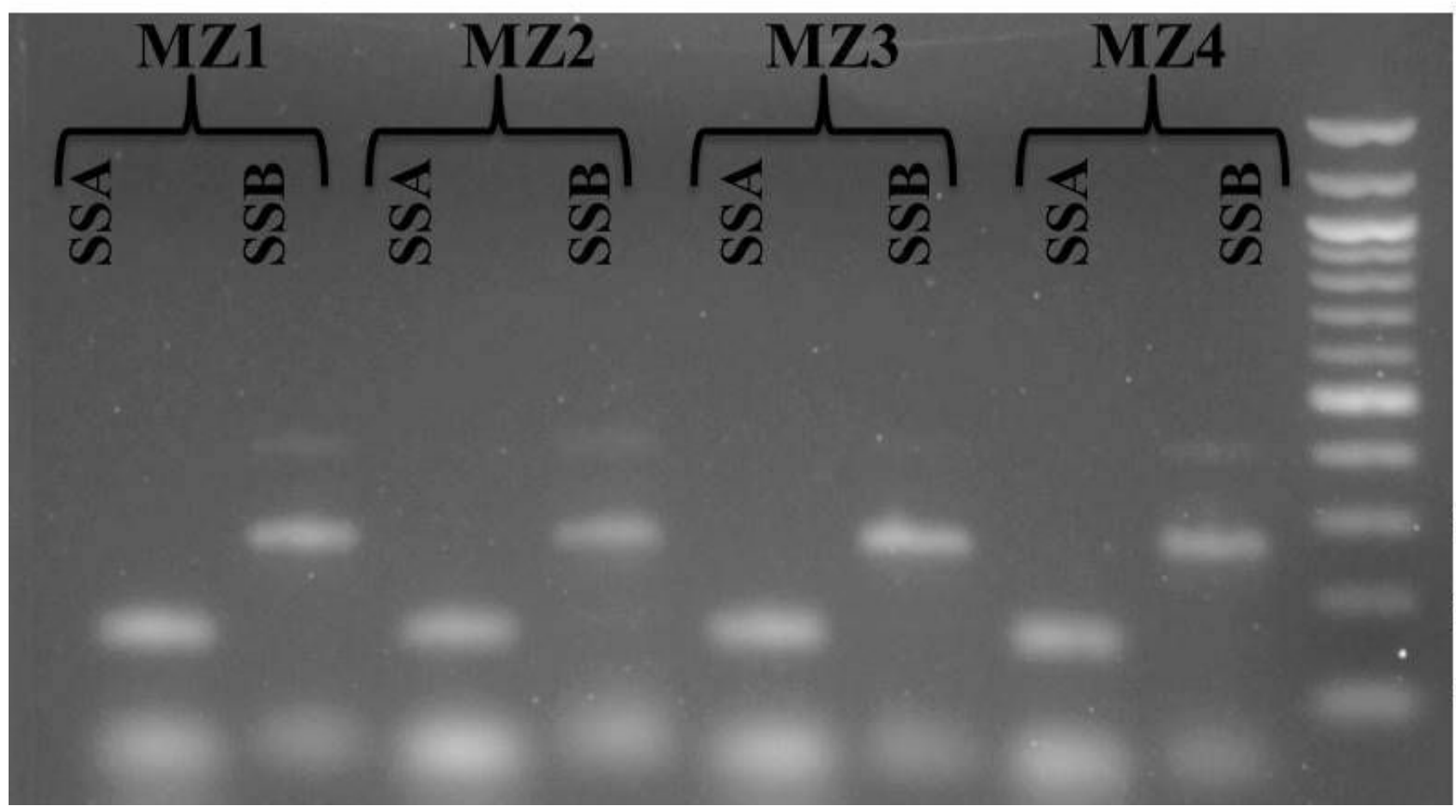

300 bp 200 bp 100 bp

Figure 3 
Agarose gels showing the results of direct PCR on nasal microbiome samples using staphostatin $A$ and staphostatin B primers. MZ1 to MZ4 are four representative nasal microbiome samples. SSA: staphostatin A primers; SSB: staphostatin B primers

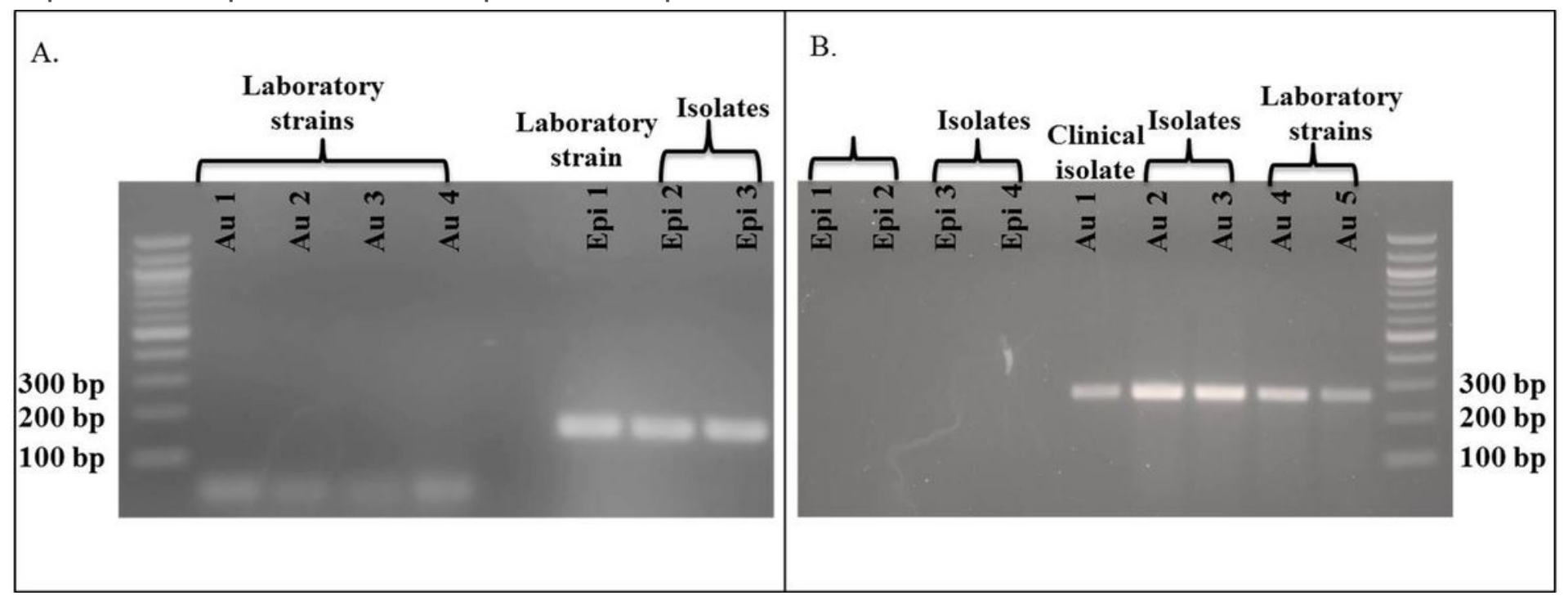

\section{Figure 4}

Agarose gels showing results of PCR performed on laboratory strains and clinical isolates. A. Results of PCR on laboratory S. aureus (Au) and S. epidermidis (Epi) strains using staphostatin A primers. B. Results of PCR on laboratory $S$. aureus and S. epidermidis strains, as well as two representative clinical $S$. aureus isolates, using staphostatin B primers 


\begin{tabular}{|c|c|c|c|c|c|c|c|c|c|}
\hline \multirow{2}{*}{$\begin{array}{c}\text { Nasal } \\
\text { swab }\end{array}$} & \multicolumn{2}{|c|}{ Direct PCR } & \multirow[t]{2}{*}{ Isolates } & \multicolumn{2}{|c|}{ Culture } & \multicolumn{2}{|c|}{ PCR } & \multirow{2}{*}{ 를 } & \\
\hline & $\mathbf{A u}$ & Epi & & Au & Epi & Au & Epi & & \\
\hline \multirow{2}{*}{ MZ1 } & \multirow{2}{*}{+} & \multirow{2}{*}{+} & MT9 & + & - & + & - & & \\
\hline & & & MT11 & - & + & - & + & & \\
\hline \multirow{3}{*}{ MZ2 } & \multirow{3}{*}{+} & \multirow{3}{*}{+} & MT12 & - & + & - & + & & \\
\hline & & & MT15 & - & + & - & + & 0 & \\
\hline & & & MT18 & - & + & - & + & & \\
\hline \multirow{2}{*}{ MZ3 } & \multirow{2}{*}{+} & \multirow{2}{*}{+} & $\mathrm{MT}$ & + & - & + & - & & \\
\hline & & & MT16 & + & - & + & - & & \\
\hline \multirow{2}{*}{ MZ4 } & \multirow{2}{*}{+} & \multirow{2}{*}{+} & MT2 & + & - & + & - & & \\
\hline & & & MT3 & - & + & - & + & & \\
\hline \multirow{2}{*}{ MZ5 } & \multirow{2}{*}{+} & \multirow{2}{*}{+} & MT21 & - & + & - & + & & \\
\hline & & & MT22 & - & + & - & + & & \\
\hline \multirow{3}{*}{ MZ6 } & \multirow{3}{*}{+} & \multirow{3}{*}{+} & MT6 & - & + & - & + & & \\
\hline & & & MT8 & + & - & + & - & & \\
\hline & & & MT10 & - & + & - & + & & \\
\hline MZ7 & + & + & MT19 & - & + & - & + & & \\
\hline MZ8 & + & + & MT20 & - & + & - & + & 0 & \\
\hline \multirow{2}{*}{ MZ9 } & \multirow{2}{*}{+} & \multirow{2}{*}{+} & MT23 & - & + & - & + & & \\
\hline & & & MT24 & + & - & + & - & & \multirow{7}{*}{$\begin{array}{l}\text { Both } S \text {. aureus and } S \text {. epidermidis were detected } \\
\text { by direct PCR and culture methods. } \\
\text { S. aureus was detected only by direct PCR, } \\
\text { while } S \text {. epidermidis was detected by direct PCR } \\
\text { and culture methods. } \\
\text { S. epidermidis was detected only by direct PCR, } \\
\text { while } S \text {. aureus was detected by direct PCR and } \\
\text { culture methods }\end{array}$} \\
\hline \multirow{2}{*}{ MZ10 } & \multirow{2}{*}{+} & \multirow{2}{*}{+} & MT5 & - & + & - & + & & \\
\hline & & & MT17 & + & - & + & - & 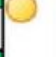 & \\
\hline & & & MT13 & - & + & - & + & & \\
\hline ML1 & + & + & MT14 & - & + & - & + & & \\
\hline MZ12 & + & + & MT1 & + & - & + & - & م & \\
\hline & & $\top$ & MT4 & + & - & + & - & & \\
\hline
\end{tabular}

Figure 5

Results of PCR directly performed on extracted community DNA from nasal samples vs. PCR performed on individual cultured isolates. MZ1-MZ12 are nasal microbiome samples (swabs), each from a different individual. MT1 to MT24 are representatives of morphologically different colonies from the above swabs. $\mathrm{Au}=$ Staphylococcus aureus; Epi = Staphylococcus epidermidis

\section{Supplementary Files}

This is a list of supplementary files associated with this preprint. Click to download.

- supplement1.pdf

- supplement2.docx 